\title{
BRAF V600E-specific immunohistochemistry reveals low mutation rates in biliary tract cancer and restriction to intrahepatic cholangiocarcinoma
}

Benjamin Goeppert ${ }^{1}$, Lena Frauenschuh ${ }^{1}$, Marcus Renner ${ }^{1}$, Stephanie Roessler ${ }^{1}$, Albrecht Stenzinger ${ }^{1}$, Frederick Klauschen ${ }^{2}$, Arne Warth ${ }^{1}$, Monika Nadja Vogel ${ }^{3}$, Arianeb Mehrabi ${ }^{4}$, Mohammadreza Hafezi ${ }^{4}$, Katja Boehmer ${ }^{5}$, Andreas von Deimling ${ }^{5,6}$, Peter Schirmacher ${ }^{1}$, Wilko Weichert ${ }^{1}$ and David Capper ${ }^{5,6}$

${ }^{1}$ Institute of Pathology, University Hospital Heidelberg, Heidelberg, Germany; ${ }^{2}$ Institute of Pathology, Charité Universitätsmedizin Berlin, Berlin, Germany; ${ }^{3}$ Department of Neuroradiology, University Hospital

Heidelberg, Heidelberg, Germany; ${ }^{4}$ Department of General Visceral and Transplantation Surgery, University Hospital Heidelberg, Heidelberg, Germany; ${ }^{5}$ Department of Neuropathology, University Hospital Heidelberg, Heidelberg, Germany and ${ }^{6}$ Clinical Cooperation Unit Neuropathology, German Cancer Research Center

(DKFZ), Heidelberg, Germany

\begin{abstract}
$B R A F$ mutations have emerged as an important predictive biomarker for metastasized melanoma. Other types of cancer may also benefit from BRAF mutation-targeted therapies. In biliary tract cancer, reported BRAF mutation rates are highly controversial, ranging from 0 to $33 \%$ in adenocarcinoma of the gallbladder and 0 to $22 \%$ in cholangiocarcinoma. We here analyzed tissue microarrays of a large cohort of biliary tract cancer $(n=377)$ including 159 intrahepatic cholangiocarcinomas, 149 extrahepatic cholangiocarcinomas, and 69 adenocarcinomas of the gallbladder for BRAF V600E mutation using a highly sensitive immunohistochemical screening approach implementing the BRAF V600E protein-specific antibody VE1. All VE1-positive cases as well as 42 VE1-negative cases were additionally analyzed by Sanger sequencing. In total, only 5 VE1-positive cases were detected (5/377; 1\%). BRAF V600E mutation was confirmed by direct sequencing in all cases. All 5 mutated cases were intrahepatic cholangiocarcinomas $(5 / 159 ; 3 \%)$. None of the extrahepatic cholangiocarcinomas and adenocarcinomas of the gallbladder were VE1 positive. Apart from the subtype restriction of BRAF V600E mutation to intrahepatic cholangiocarcinoma and a female predominance (4 female, 1 male), no significant correlation with clinicopathological data and patient outcome was detected. In conclusion, we demonstrate that BRAF V600E mutation is a rare event in biliary tract cancer, accounting for only $1 \%$ of all subtypes, and is restricted to intrahepatic cholangiocarcinoma. In addition, we demonstrate that VE1 immunohistochemistry is a feasible approach to routinely screen for BRAF V600E mutation in biliary tract cancer patients, thereby facilitating the detection of rare patients who may benefit from BRAF mutation-targeted therapies.

Modern Pathology (2014) 27, 1028-1034; doi:10.1038/modpathol.2013.206; published online 6 December 2013
\end{abstract}

Keywords: biliary tract cancer; BRAF; cholangiocarcinoma; immunohistochemistry; VE1; V600E

Biliary tract cancers are a diverse group of tumors that arise from the biliary tract epithelium. Biliary tract cancers can be divided into three major clinical

Correspondence: Dr D Capper, MD, Department of Neuropathology, Institute of Pathology, Ruprecht-Karls-University Heidelberg, Im Neuenheimer Feld 224, 69120 Heidelberg, Germany.

E-mail: david.capper@med.uni-heidelberg.de

Received 21 June 2013; accepted 24 September 2013; published online 6 December 2013 phenotypes: cholangiocarcinomas of intrahepatic and extrahepatic origin and adenocarcinomas of the gallbladder. In the clinical context, biliary tract cancers are often treated as one disease, although there is evidence of genetic heterogeneity as well as differences in clinical behavior. ${ }^{1}$ Most patients with biliary tract cancer present with unresectable or metastatic disease. Despite systemic chemotherapy, prognosis remains poor and to date there are no moleculartargeted therapies tailored to biliary tract cancer. ${ }^{2}$ 
The RAS/RAF/MEK/ERK mitogen-activated protein (MAP) kinase pathway regulates cellular proliferation, survival, and migration, and is aberrantly activated in the majority of solid tumors. Mutation of the $B R A F$ gene is one mechanism of constitutive activation and occurs in many human cancers including cutaneous melanoma, papillary thyroid carcinoma, borderline ovarian carcinoma, pleomorphic xanthoastrocytoma, colorectal carcinoma, non-small cell lung carcinoma, and hairy cell leukemia, among others. ${ }^{3-6}$ The most common BRAF mutation results in a single aminoacid substitution of valine for glutamic acid at residue 600 (V600E). Early publications referred to codon 600 mutations as codon 599 mutations. In 2003, the $B R A F$ sequence was updated with the insertion of $3 \mathrm{bp}$ in the coding sequence, resulting in a new number of the hotspot codon (600 instead of 599). For metastatic melanoma, small-molecule inhibitors of BRAF V600E protein have demonstrated clinical activity and have rapidly changed standard treatment of $B R A F$ mutated melanoma patients. $^{7-9}$ Similarly, these targeted therapies might also have antitumor activity in various other $B R A F$-mutated neoplasias. ${ }^{10-14}$ Accurate and rapid detection of $B R A F$ mutations in metastatic melanoma is therefore essential for optimal patient care and may possibly be also required in other tumor entities in the near future.

An analysis of available data on $B R A F$ V600E and non-V600E mutations revealed that $B R A F$ mutation frequencies are highly controversial in biliary tract cancers ranging from 0 to $33 \%$ for $B R A F$ V600E (see Table 1). As most studies with high $B R A F$ mutation rates were performed on European cohorts, a recent review raised the question of whether these discordant results represent a regional difference in the genetics of biliary tract cancer. ${ }^{2}$

In this study, we investigated a large collection of biliary tract cancers for $B R A F$ V600E mutations using a novel mutation-specific antibody (clone VE1) that specifically binds to BRAF V600E-mutated protein that is in general located in the cytoplasm. VE1 immunohistochemistry has proven to be highly sensitive and specific for various tumor types, including melanoma, ${ }^{15-19}$ thyroid carcinoma, ${ }^{20,21}$ colorectal carcinoma, ${ }^{22,23}$ ganglioglioma, ${ }^{24}$ and hairy cell leukemia, ${ }^{25}$ among others.

The large numbers of the cohort investigated in this study should allow (1) to gain a more solid data basis for estimating the frequency of $B R A F \mathrm{~V} 600 \mathrm{E}$ mutation in biliary tract cancer, thereby partly resolving the discrepancy of the previously reported mutation rates, and (2) detecting associations of $B R A F$ mutation status with subtypes and possibly other clinicopathological factors. Furthermore, we present a feasible approach to screen biliary tract cancer specimen for $B R A F$ V600E mutation.

\section{Materials and methods}

\section{Clinicopathological Characteristics of Biliary Tract Cancer Patients}

Tissue samples from 377 patients (median age 64.1 years) who underwent bile duct and/or liver surgery at the University Hospital Heidelberg between 1995 and 2010 were included in this study. Only patients with primary adenocarcinomas of the biliary tract and without other known malignancies at the time of diagnosis were included. Biliary tract cancers consisted of 159 intrahepatic cholangiocarcinomas, 149 extrahepatic cholangiocarcinomas (106 perihilar and 43 distal), and 69 adenocarcinomas of the gallbladder. None of the patients received radioand/or chemotherapy before surgery. Tumors were restaged according to the 7 th TNM Classification of Malignant Tumors and classified after the World Health Organization (WHO) tumor classification system (WHO Classification of Tumours of the Digestive System, 4th edn, 2010) by two experienced pathologists (BG and WW). A summary of clinicopathological data is given in Table 2. The use of the tissues for this study was approved by the institutional ethics committee (206/05).

\section{Tissue Microarray Construction}

From all 377 biliary tract cancers, $3 \mu \mathrm{m}$ sections were cut and stained with H\&E. Representative areas

Table 1 Overview of studies that have examined $B R A F$ mutation status in biliary tract cancer

\begin{tabular}{|c|c|c|c|c|}
\hline Study & $\begin{array}{c}\text { Adenocarcinoma } \\
\text { of the gallbladder } \\
\text { BRAF V600E }\end{array}$ & $\begin{array}{c}\text { Cholangiocarcinoma } \\
\text { BRAF V600E }\end{array}$ & $\begin{array}{c}\text { Intrahepatic } \\
\text { cholangiocarcinoma } \\
\text { BRAF V600E }\end{array}$ & $\begin{array}{c}\text { Extrahepatic } \\
\text { cholangiocarcinoma } \\
\text { BRAF V600E }\end{array}$ \\
\hline Tannapfel et $a l^{29}$ & Not investigated & $11 / 69(13 \%)^{\mathrm{a}}$ & CC subtype not specified & CC subtype not specified \\
\hline Saetta et al ${ }^{30}$ & $7 / 21(33 \%)$ & Not investigated & Not investigated & Not investigated \\
\hline Goldenberg et al ${ }^{31}$ & $0 / 37(0 \%)$ & $0 / 25(0 \%)$ & $0 / 10(0 \%)$ & $0 / 15(0 \%)$ \\
\hline Borger et $a l^{32}$ & $0 / 25(0 \%)$ & $1 / 62(2 \%)$ & $1 / 40(3 \%)$ & $0 / 22(0 \%)$ \\
\hline Andersen et al ${ }^{28}$ & Not investigated & 1/69 (1\%) & CC subtype not specified & CC subtype not specified \\
\hline Sia et $a l^{27}$ & Not investigated & $3 / 141(2 \%)^{b}$ & $3 / 141(2 \%)^{b}$ & Not investigated \\
\hline Voss et $a l^{26}$ & Not investigated & $2 / 94(2 \%)$ & $2 / 67(3 \%)$ & $0 / 27(0 \%)$ \\
\hline This study & $0 / 69(0 \%)$ & $5 / 308(2 \%)$ & $5 / 159(3 \%)$ & $0 / 149(0 \%)$ \\
\hline
\end{tabular}

Abbreviation: CC, cholangiocarcinoma.

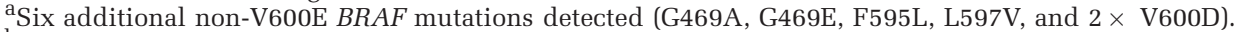

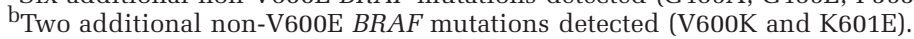


were marked by two experienced pathologists (BG and WW). For each case, tumor tissue cores $(1.0 \mathrm{~mm}$ diameter) from the selected representative tumor areas were punched out of the sample tissue blocks and embedded into a new paraffin array block using a tissue microarrayer (Beecher Instruments, Woodland, CA, USA).

\section{Immunohistochemistry}

Tissue microarray blocks were cut to $3 \mu \mathrm{m}$ sections. BRAF V600E protein automated immunohistochemistry (clone VE1) was performed as previously described using the Ventana UltraView chemistry. ${ }^{25}$ Scoring of immunostaining results was performed by two experienced observers (BG and DC) blinded to all clinicopathological and genetic data. Immunoreaction was scored positive, when viable tumor cells showed a nonambiguous cytoplasmic staining for VE1. A faint diffuse staining, any type of isolated nuclear staining, weak staining of single interspersed cells, or staining of monocytes/macrophages was not considered positive.

\section{PCR Amplification and Direct Sequencing}

In all selected cases (all immunopositive tumors and 42 randomly chosen immunohistochemistry-negative control intrahepatic cholangiocarcinoma cases), we performed direct sequencing of $B R A F$ exon 15 as previously described. ${ }^{3}$ In addition, we tested $B R A F$ V600E-mutated cases for KRAS hotspot mutations by sequencing (complete exon 1) using standard primers. Microdissection was performed if tumor content was $<60 \%$.

\section{Statistical Analyses}

Statistical analyses were performed with the statistical computing environment $\mathrm{R}$ version 2.15.1. Correlation analyses of BRAF V600E status with clinicopathological variables were assessed with Fisher's exact test. Univariate survival analysis was performed for overall survival by generation of Kaplan-Meier curves. Significance of differences between the groups was assessed using the log-rank test. The $P$-values of $<0.05$ were considered significant.

\section{Results}

Frequency of BRAF V600E Mutation in Biliary Tract Cancer and Correlation with Clinicopathological Data

Our immunohistochemical screen of 377 biliary tract cancers revealed 5 VE1-positive cases (1\%). Immunoreaction was cytoplasmic, moderate to strong, and homogenous, staining all observable viable tumor cells in all five cases. Analysis of biliary tract
Table 2 Clinicopathological data of biliary tract cancer cohort with complete clinicopathological data and correlation with the $B R A F$ V600E status

\begin{tabular}{|c|c|c|c|}
\hline & & Number (\%) & \\
\hline & Number (\%) & $\begin{array}{l}\text { BRAF } \\
\text { V600E }\end{array}$ & $\begin{array}{l}\text { Fisher's } \\
\text { exact test }\end{array}$ \\
\hline BTC patients & $377(100 \%)$ & $5(1 \%)$ & NS \\
\hline \multicolumn{4}{|l|}{ Age } \\
\hline 64-92 Years & $187(50 \%)$ & $2(1 \%)$ & NS \\
\hline 31-64 Years & $190(50 \%)$ & $3(2 \%)$ & NS \\
\hline \multicolumn{4}{|l|}{ Sex } \\
\hline M & $190(50 \%)$ & $1(1 \%)$ & 0.37 \\
\hline W & $187(50 \%)$ & $4(2 \%)$ & NS \\
\hline \multicolumn{4}{|l|}{$\operatorname{UICC}(\mathrm{N}=296)$} \\
\hline UICC 1 & $40(14 \%)$ & $2(5 \%)$ & NS \\
\hline UICC 2 & $75(25 \%)$ & $1(1 \%)$ & NS \\
\hline UICC 3 & $82(28 \%)$ & $0(0 \%)$ & NS \\
\hline UICC 4 & $99(33 \%)$ & $2(2 \%)$ & NS \\
\hline \multicolumn{4}{|l|}{$p T$} \\
\hline $\mathrm{T} 1$ & $80(21 \%)$ & $1(1 \%)$ & NS \\
\hline $\mathrm{T} 2$ & $148(39 \%)$ & $3(2 \%)$ & NS \\
\hline T3 & $117(31 \%)$ & $1(1 \%)$ & NS \\
\hline $\mathrm{T} 4$ & $32(9 \%)$ & $0(0 \%)$ & NS \\
\hline \multicolumn{4}{|l|}{$p N(\mathrm{~N}=286)$} \\
\hline No & $129(45 \%)$ & $2(2 \%)$ & NS \\
\hline N1 & $157(55 \%)$ & $2(1 \%)$ & NS \\
\hline \multicolumn{4}{|l|}{$M$} \\
\hline Mo & $354(94 \%)$ & $5(1 \%)$ & NS \\
\hline M1 & $23(6 \%)$ & $0(0 \%)$ & NS \\
\hline \multicolumn{4}{|l|}{$G$} \\
\hline G1 & $20(5 \%)$ & $0(0 \%)$ & NS \\
\hline G2 & $255(68 \%)$ & $4(2 \%)$ & NS \\
\hline G3 & $102(27 \%)$ & $1(1 \%)$ & NS \\
\hline \multicolumn{4}{|l|}{$L$} \\
\hline L0 & $174(46 \%)$ & $2(1 \%)$ & NS \\
\hline L1 & $203(54 \%)$ & $3(2 \%)$ & NS \\
\hline \multicolumn{4}{|l|}{$V$} \\
\hline V0 & $275(73 \%)$ & $3(1 \%)$ & NS \\
\hline V1 & $102(27 \%)$ & $2(2 \%)$ & NS \\
\hline \multicolumn{4}{|l|}{$P n$} \\
\hline Pno & $294(78 \%)$ & $5(2 \%)$ & NS \\
\hline Pn1 & $83(22 \%)$ & $0(0 \%)$ & NS \\
\hline \multicolumn{4}{|c|}{ Biliary tract cancer subgroups } \\
\hline $\begin{array}{l}\text { Intrahepatic } \\
\text { cholangiocarcinoma }\end{array}$ & $159(42 \%)$ & $5(3 \%)$ & 0.01 \\
\hline $\begin{array}{l}\text { Extrahepatic } \\
\text { cholangiocarcinoma }\end{array}$ & $149(40 \%)$ & $0(0 \%)$ & NS \\
\hline $\begin{array}{l}\text { Adenocarcinomas } \\
\text { of the gallbladder }\end{array}$ & $69(18 \%)$ & $0(0 \%)$ & NS \\
\hline \multicolumn{4}{|l|}{ Histology } \\
\hline Ductal & 308 (82\%) & $4(1 \%)$ & NS \\
\hline Papillary & 25 (7\%) & $1(4 \%)$ & NS \\
\hline Mucinous & $10(3 \%)$ & $0(0 \%)$ & NS \\
\hline Intestinal & $10(3 \%)$ & $0(0 \%)$ & NS \\
\hline Other & $24(6 \%)$ & $0(0 \%)$ & NS \\
\hline
\end{tabular}


cancer subtypes demonstrated that all 5 positive cases were intrahepatic cholangiocarcinomas (5/ 159, 3\%), whereas all extrahepatic cholangiocarcinomas $(n=149)$ and adenocarcinomas of the gallbladder $(n=69)$ were VE1 negative (Figure 1 and Table 2). BRAF V600E mutation was confirmed by Sanger sequencing in all five cases. In 42 randomly chosen VE1 immunonegative intrahepatic cholangiocarcinoma control cases, no BRAF V600E and no other exon 15 hotspot mutation was detected by sequencing. Among the $B R A F$-mutated cases, no
KRAS mutations were detected (data not shown). Despite the low number of detected mutations, the subtype restriction of $B R A F$ V600E mutation to intrahepatic cholangiocarcinoma was statistically significant $(P=0.01)$. A statistically nonsignificant female predominance (4 female, 1 male; $P=0.37$ ), but no further correlation of cases harboring $B R A F$ V600E mutation with clinicopathological data or specific etiology (such as primary sclerosing cholangitis, HCV infection, Caroli disease), was detected (Table 2).
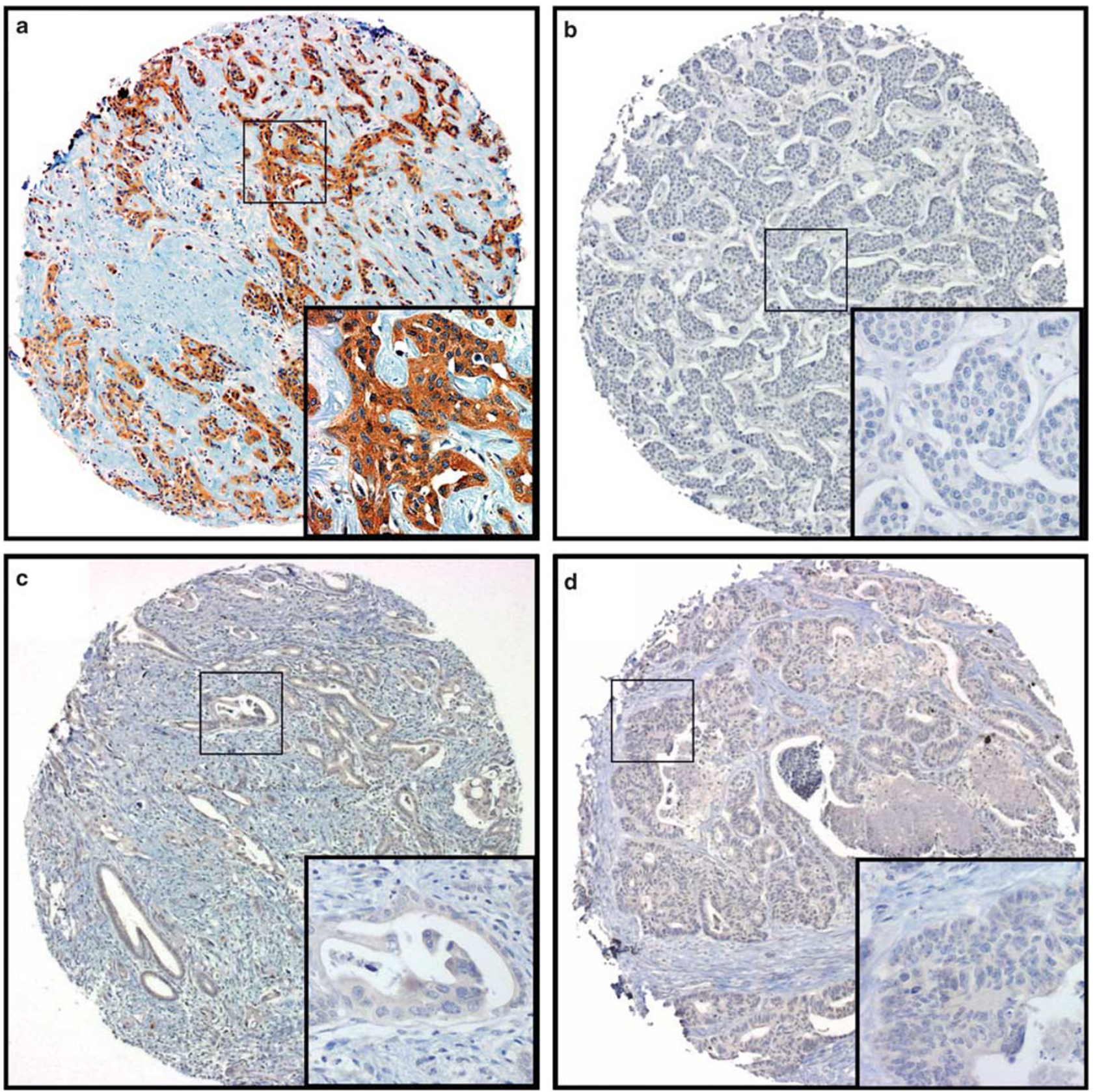

Figure 1 Representative micrographs of BRAF V600E-specific immunohistochemistry (clone VE1) of biliary tract cancer tissue microarray. (a) Strong homogenous cytoplasmic immunoreactivity in an intrahepatic cholangiocarcinoma. (b) Different intrahepatic cholangiocarcinoma with negative VE1 staining. (c) All extrahepatic cholangiocarcinomas $(n=149)$ were negative for VE1. (d) All adenocarcinoma of the gallbladder $(n=69)$ were negative for VE1. Magnification 50-fold, inset 200-fold. 
Correlation of BRAF V600E Mutation Status with Survival of Intrahepatic Cholangiocarcinoma Patients

Among intrahepatic cholangiocarcinoma, patients with BRAF V600E-mutated tumors did not show an altered overall survival probability when compared with patients with BRAF wild-type tumors (Figure 2; $P=0.38$ ).

\section{Discussion}

This analysis of a comprehensive biliary tract cancer cohort demonstrates that BRAF V600E mutation is a rare event in biliary tract cancer, accounting for only $1 \%$ of all cases $(n=377)$. Our findings are in line with recently published smaller series detecting $B R A F$ mutations in $1 \%$ to $4 \%$ of evaluated cases. ${ }^{26-28}$ We cannot confirm the exceedingly high mutation rates detected in early BRAF studies of European cohorts, ${ }^{29,30}$ and conclude that the previously assumed regional difference in the genetics of biliary tract cancer in Europe versus the Americas is not existent. $^{2}$ Our data further demonstrate that $B R A F$ mutations are restricted to intrahepatic cholangiocarcinomas and account for $3 \%$ in this subgroup. Comparing these results with earlier studies demonstrates that this restriction to intrahepatic cholangiocarcinoma is also evident in most preceding studies, although the case numbers were previously too low for further statistical conclusions (Table 1). A single early study reported several BRAF V600Emutated cases among adenocarcinomas of the gallbladder. ${ }^{30}$ Our data and the data of two other groups implementing diverse methods for mutation detection weaken the generalizability of this early observation in adenocarcinomas of the gallbladder. ${ }^{31,32}$

Most intrahepatic cholangiocarcinomas are diagnosed at an unresectable stage, for which only palliative chemotherapy remains as therapeutic option. ${ }^{1,2}$ Thus, detection of a potentially treatable $B R A F$ V600E mutation may represent an additional treatment option for these patients. The exclusive detection of BRAF V600E mutation in intrahepatic

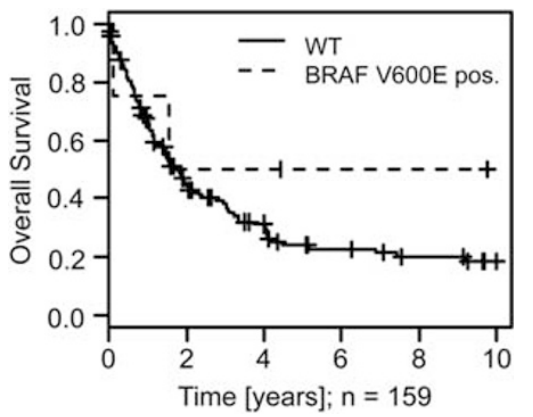

Figure 2 Overall survival probability in intrahepatic cholangiocarcinoma patients in correlation with BRAF V600E status. Kaplan-Meier curves show no difference in overall survival of patients in correlation with BRAF V600E status in intrahepatic cholangiocarcinoma $(P=0.38)$. The $P$-values were calculated with a log-rank test. cholangiocarcinoma also has practical diagnostic implications, because the screening for BRAF V600E mutation in biliary tract cancer could be narrowed to this subtype, thereby substantially lowering the number of cases to be screened. Furthermore, our observation adds to the growing notion of a distinct molecular separation of intrahepatic and extrahepatic cholangiocarcinoma ${ }^{1,2}$ as also recently observed for mutations of isocytrate dehydrogenase 1 and $2 .^{32}$

Correlation of BRAF V600E mutation status with survival of intrahepatic cholangiocarcinoma patients did not show any significant difference. However, this conclusion is of limited validity because of the low number of observed mutated cases. In other types of cancer, BRAF mutations have shown varying association with patient mortality, but the effect is generally not independent of other tumor features, and thus BRAF status currently does not give definite prognostic implications for most tumor types. ${ }^{33,34}$

Implementation of VE1 immunohistochemistry for the prediction of a BRAF V600E mutation has been investigated by various groups and for various types of cancer. ${ }^{15-21,23-25}$ VE1 immunohistochemistry has the advantage of being rapid and relatively inexpensive, while the sensitivity and specificity are comparable to DNA-based methods. For pathologists, an additional well-appreciated advantage is the in situ mutation detection that provides information on the mutated cell population, as well as an additional visual sample verification that a faceless DNA sample does not offer. The utilization of such a technique seems particularly feasible when the expected rate of mutations is as low as in intrahepatic cholangiocarcinoma, and routine implementation of more work-intense methods such as direct sequencing is less attractive. Use of VE1 to screen for low-frequency $B R A F$ V600E mutations has previously been demonstrated for primary lung adenocarcinoma and for unselected brain metastases of various histologies. ${ }^{35,36}$

A clear disadvantage is that the immunohistochemical mutation analysis is limited to $B R A F$ V600E. This is of highest relevance in melanoma, where depending on tumor genesis (especially sun exposure) up to $25 \%$ of $B R A F$ mutations may be non-V600E. ${ }^{34}$ In biliary tract cancer, the vast majority of $B R A F$ mutations reported to date were of the V600E type, with the limitation that several studies used DNA-based methods that are only able to detect a limited spectrum of BRAF mutations (eg, the Prism SNaPshot Multiplex system that can detect V600A, E, G, and $\mathrm{M}^{32}$ ). In two previously analyzed cholangiocarcinoma cohorts, tumors with BRAF mutations other than V600E have been reported that may potentially also respond to targeted therapy. For BRAF V600K (detected in one intrahepatic cholangiocarcinoma by Sia et $a l^{27}$ ) response of melanoma metastases has been observed in a phase II trial, ${ }^{37}$ and for BRAF V600D (detected in two cholangiocarcinomas not otherwise specified by Tannapfel et $a l^{29}$ ) in vitro inhibitor 
activity was shown in a melanoma cell line. ${ }^{38}$ In single instances, other rare $B R A F$ mutations were detected (eg, BRAF K601E) for which data on potential clinical activity of $B R A F$ inhibitors are not available. VE1 immunohistochemistry does not detect V600K, V600D, or other rare mutations, ${ }^{15,16}$ and would thus fail to identify these potential candidates for targeted therapy. In our collection of 42 VE1-negative intrahepatic cholangiocarcinomas, we did not observe any non-V600E mutations, indicating that they altogether likely represent rare events in intrahepatic cholangiocarcinoma.

In conclusion, we demonstrate that VE1 immunohistochemistry is a feasible and valid approach for screening for $B R A F \mathrm{~V} 600 \mathrm{E}$ mutation in biliary tract cancer. In biliary tract cancer, $B R A F$ V600E mutation is restricted to intrahepatic cholangiocarcinomas, and screening of extrahepatic cholangiocarcinomas and adenocarcinomas of the gallbladder could therefore likely be omitted. Considering that $\sim 70000$ new cases of intrahepatic cholangiocarcinoma are diagnosed globally every year, $\sim 2000$ to 3000 patients are expected to harbor $B R A F$ V600Emutated tumors. ${ }^{27}$ These patients may benefit from targeted therapies, and $B R A F$ mutation screening approaches-like the one presented in this studyshould be evaluated for design of prospective trials for such low $B R A F$ mutation frequency populations.

\section{Acknowledgments}

We thank John Moyers (Institute of Pathology, University of Heidelberg) for excellent technical assistance. We thank the NCT tissue bank Heidelberg for its support (tissue banking, tissue microarray manufacturing, and technical assistance). This work was supported by grants from the Deutsche Forschungsgemeinschaft (DFG) to P Schirmacher (SFB/TRR77).

\section{Disclosure/conflict of interest}

Under a licensing agreement between Ventana Medical Systems, Tucson, Arizona, and the German Cancer Research Center, DC and AvD are entitled to a share of royalties received by the German Cancer Research Center on the sales of VE1 antibody. The terms of this arrangement are being managed by the German Cancer Research Center in accordance with its conflict of interest policies.

\section{References}

1 Blechacz B, Komuta M, Roskams T, et al. Clinical diagnosis and staging of cholangiocarcinoma. Nat Rev Gastroenterol Hepatol 2011;8:512-522.

2 Hezel AF, Deshpande V, Zhu AX. Genetics of biliary tract cancers and emerging targeted therapies. J Clin Oncol 2010;28:3531-3540.
3 Schindler G, Capper D, Meyer J, et al. Analysis of BRAF V600E mutation in 1,320 nervous system tumors reveals high mutation frequencies in pleomorphic xanthoastrocytoma, ganglioglioma and extra-cerebellar pilocytic astrocytoma. Acta Neuropathol 2011;121: 397-405.

4 Michaloglou C, Vredeveld LC, Mooi WJ, et al. BRAF(E600) in benign and malignant human tumours. Oncogene 2008;27:877-895.

5 Davies H, Bignell GR, Cox C, et al. Mutations of the BRAF gene in human cancer. Nature 2002;417:949-954.

6 Arkenau HT, Kefford R, Long GV. Targeting BRAF for patients with melanoma. Br J Cancer 2011;104:392-398.

7 Flaherty KT, Puzanov I, Kim KB, et al. Inhibition of mutated, activated BRAF in metastatic melanoma. N Engl J Med 2010;363:809-819.

8 Chapman PB, Hauschild A, Robert C, et al. Improved survival with vemurafenib in melanoma with BRAF V600E mutation. N Engl J Med 2011;364:2507-2516.

9 Hauschild A, Grob JJ, Demidov LV, et al. Dabrafenib in BRAF-mutated metastatic melanoma: a multicentre, open-label, phase 3 randomised controlled trial. Lancet 2012;380:358-365.

10 Rush S, Foreman N, Liu A. Brainstem ganglioglioma successfully treated with vemurafenib. J Clin Oncol 2013;31:e159-e160.

11 Kim K, Cabanillas M, Lazar AJ, et al. Clinical responses to vemurafenib in patients with metastatic papillary thyroid cancer harboring V600EBRAF mutation. Thyroid 2013;23:1277-1283.

12 Falchook GS, Long GV, Kurzrock R, et al. Dabrafenib in patients with melanoma, untreated brain metastases, and other solid tumours: a phase 1 dose-escalation trial. Lancet 2012;379:1893-1901.

13 Dietrich S, Glimm $\mathrm{H}$, Andrulis $\mathrm{M}$, et al. BRAF inhibition in refractory hairy-cell leukemia. N Engl J Med 2012;366:2038-2040.

14 Andrulis M, Lehners N, Capper D, et al. Targeting the BRAF V600E mutation in multiple myeloma. Cancer Discov 2013;3:862-869.

15 Skorokhod A, Capper D, von Deimling A, et al. Detection of BRAF V600E mutations in skin metastases of malignant melanoma by monoclonal antibody VE1. J Am Acad Dermatol 2012;67:488-491.

16 Long GV, Wilmott JS, Capper D, et al. Immunohistochemistry is highly sensitive and specific for the detection of V600E BRAF mutation in melanoma. Am J Surg Pathol 2013;37:61-65.

17 Colomba E, Helias-Rodzewicz Z, Von Deimling A, et al. Detection of BRAF p.V600E mutations in melanomas: comparison of four methods argues for sequential use of immunohistochemistry and pyrosequencing. J Mol Diagn 2013;15:94-100.

18 Lade-Keller J, Kristensen LS, Riber-Hansen R, et al. A role for immunohistochemical detection of BRAF V600E prior to BRAF-inhibitor treatment of malignant melanoma? J Clin Pathol 2013;66:723-725.

19 Busam KJ, Hedvat C, Pulitzer M, et al. Immunohistochemical analysis of BRAF(V600E) expression of primary and metastatic melanoma and comparison with mutation status and melanocyte differentiation antigens of metastatic lesions. Am J Surg Pathol 2013; 37:413-420.

20 Koperek O, Kornauth C, Capper D, et al. Immunohistochemical detection of the BRAF V600E-mutated protein in papillary thyroid carcinoma. Am J Surg Pathol 2012;36:844-850. 
21 Bullock M, O’Neill C, Chou A, et al. Utilization of a MAB for BRAF(V600E) detection in papillary thyroid carcinoma. Endocr Relat Cancer 2012;19: 779-784.

22 Toon CW, Walsh MD, Chou A, et al. BRAFV600E immunohistochemistry facilitates universal screening of colorectal cancers for Lynch Syndrome. Am J Surg Pathol 2013;37:1592-1602.

23 Capper D, Voigt A, Bozukova G, et al. BRAF V600Especific immunohistochemistry for the exclusion of Lynch syndrome in MSI-H colorectal cancer. Int J Cancer 2013;133:1624-1630.

24 Koelsche C, Wohrer A, Jeibmann A, et al. Mutant BRAF V600E protein in ganglioglioma is predominantly expressed by neuronal tumor cells. Acta Neuropathol 2013;125:891-900.

25 Andrulis M, Penzel R, Weichert W, et al. Application of a BRAF V600E mutation-specific antibody for the diagnosis of hairy cell leukemia. Am J Surg Pathol 2012;36:1796-1800.

26 Voss JS, Holtegaard LM, Kerr SE, et al. Molecular profiling of cholangiocarcinoma shows potential for targeted therapy treatment decisions. Hum Pathol 2013;44:1216-1222.

27 Sia D, Hoshida Y, Villanueva A, et al. Integrative molecular analysis of intrahepatic cholangiocarcinoma reveals 2 classes that have different outcomes. Gastroenterology 2013;144:829-840.

28 Andersen JB, Spee B, Blechacz BR, et al. Genomic and genetic characterization of cholangiocarcinoma identifies therapeutic targets for tyrosine kinase inhibitors. Gastroenterology 2012;142:e15.

29 Tannapfel A, Sommerer F, Benicke M, et al. Mutations of the BRAF gene in cholangiocarcinoma but not in hepatocellular carcinoma. Gut 2003;52:706-712.
30 Saetta AA, Papanastasiou P, Michalopoulos NV, et al. Mutational analysis of BRAF in gallbladder carcinomas in association with K-ras and p53 mutations and microsatellite instability. Virchows Arch 2004;445: 179-182.

31 Goldenberg D, Rosenbaum E, Argani P, et al. The V599E BRAF mutation is uncommon in biliary tract cancers. Mod Pathol 2004;17:1386-1391.

32 Borger DR, Tanabe KK, Fan KC, et al. Frequent mutation of isocitrate dehydrogenase (IDH)1 and IDH2 in cholangiocarcinoma identified through broadbased tumor genotyping. Oncologist 2012;17:72-79.

33 Xing M, Alzahrani AS, Carson KA, et al. Association between BRAF V600E mutation and mortality in patients with papillary thyroid cancer. JAMA 2013;309:1493-1501.

34 Long GV, Menzies AM, Nagrial AM, et al. Prognostic and clinicopathologic associations of oncogenic BRAF in metastatic melanoma. J Clin Oncol 2011;29:1239-1246.

35 Ilie M, Long E, Hofman V, et al. Diagnostic value of immunohistochemistry for the detection of the BRAFV600E mutation in primary lung adenocarcinoma Caucasian patients. Ann Oncol 2013;24:742-748.

36 Capper D, Berghoff AS, Magerle M, et al. Immunohistochemical testing of BRAF V600E status in 1,120 tumor tissue samples of patients with brain metastases. Acta Neuropathol 2012;123:223-233.

37 Long GV, Trefzer U, Davies MA, et al. Dabrafenib in patients with Val600Glu or Val600Lys BRAF-mutant melanoma metastatic to the brain (BREAK-MB): a multicentre, open-label, phase 2 trial. Lancet Oncol 2012;13:1087-1095.

38 Gentilcore G, Madonna G, Mozzillo N, et al. Effect of dabrafenib on melanoma cell lines harbouring the BRAF(V600D/R) mutations. BMC Cancer 2013;13:17. 